\title{
Landau-Kleffner syndrome evolving to electrical status epilepticus: a case report illustrating clinical heterogeniety
}

\author{
Jayantee Kalita ${ }^{1}$, Mamta B. Singh ${ }^{1}$, Usha K. Misra ${ }^{1}$, Birendra K. Das ${ }^{2}$ \\ Departments of ${ }^{1}$ Neurology and ${ }^{2}$ Nuclear Medicine Sanjay Gandhi \\ Post Graduate Institute of Medical Sciences Lucknow, India
}

\begin{abstract}
A child with Landau-Kleffner syndrome who changed to continuous spike wave discharges both during sleep as well as awake is reported in this communication. A 4.5-year-old boy developed rapidly progressive mixed aphasia with relative preservation of writing and drawing skills. Electroencephalogram showed 2-2.5 Hz spike wave discharges more marked during slow wave sleep. Single photon emission tomography revealed hypoperfusion of the left frontotemporoparietal area. The patient did not respond to methylprednisolone, intravenous immunoglobulin and a number of antiepileptic drugs. At 2 year follow up there was no clinical improvement and the EEG showed continuous spike wave discharges not only during sleep but also during waking. He was aphasic but had preservation of spatial intelligence. This case report highlights the severe and resistant form of Landau-Kleffner syndrome and the heterogeniety of the condition. (J Pediatr Neurol 2004; 2(3): 157-160).
\end{abstract}

Key words: Landau-Kleffner syndrome, aphasia, SPECT, EEG, electrical status epilepticus.

Correspondence: Prof. Usha Kant Misra,

Department of Neurology,

Sanjay Gandhi Post Graduate

Institute of Medical Sciences

RaeBareli Road,

Lucknow- 226 014, India.

Fax: 91-0522-2668017.

E-mail: ukmisra@sgpgi.ac.in

Received: December 19, 2003.

Revised: March 13, 2004.

Accepted: April 15, 2004

\section{Introduction}

Landau-Kleffner syndrome (LKS) is a disorder of childhood characterised by acquired aphasia, paroxysmal spike and slow waves or bitemporal spikes on electroencephalogram (EEG), easily treatable or self limiting seizures, no demonstrable brain pathology to explain behavioral symptomatology and some degree of improvement when the epileptic condition resolves (1). The first manifestation of the language disturbance often is an apparent word deafness and parents usually report a gradual inability to respond to their calls because raising their voice is ineffective. This word deafness can progress to a total unresponsiveness to oral communication followed by deterioration in expressive language and vocabulary. The children may speak in a telegraphic language but occasionally there may be jargon speech (2). In older children reading and writing abilities also deteriorate (3).

We have recently managed a patient with LKS who presented with rapidly progressive and simultaneous auditory agnosia and motor aphasia. We report this patient and review the contemporary information about the etiology of this condition.

\section{Case Report}

A 4.5-year-old boy was noted to be confused and inattentive when he got up in the morning. His parents observed that he was neither comprehending nor speaking. He refused his breakfast. He seemed unaware of the instructions given to him although he would turn to loud sounds. Mother sent him to school as usual thinking that he may be sleepy. On returning from school, the child appeared normal and remained so for five days. On the sixth day he again stopped talking and behaved awkwardly; he did not respond to loud commands although responded appropriately to gestures. He was unable to speak spontaneously but could write letters, numbers and draw simple figures, which he had 


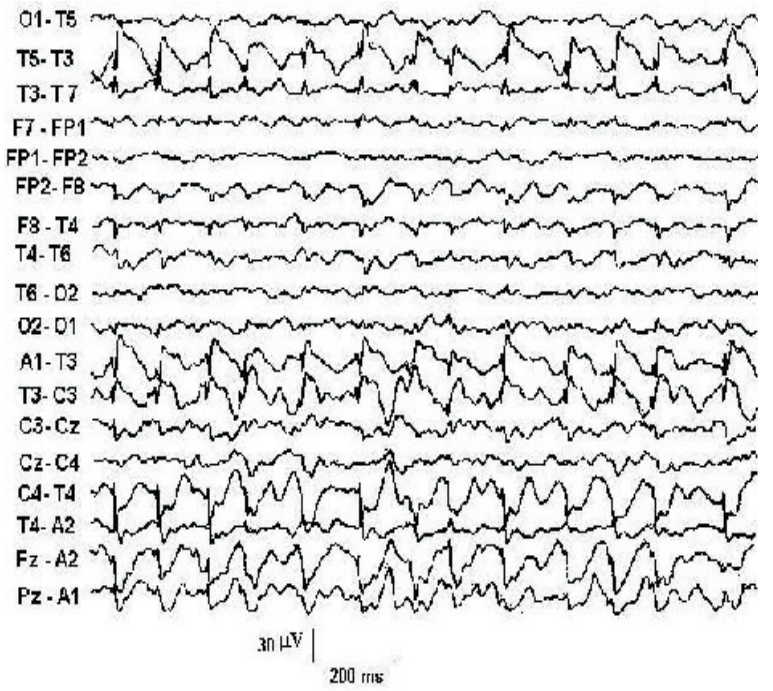

Figure 1a. EEG of the patient with Landau-Kleffner syndrome showing slow spike and slow wave discharges bilaterally which is more marked in left temporal region.

learnt earlier. There were no overt seizures during the course of his illness.

The patient was born of a non-consanguineous marriage and was a full-term vaginal delivery conducted at hospital. His mother had an uneventful prenatal course. The child cried normally after birth, weighed $3 \mathrm{~kg}$ and had a normal neonatal period. He started sitting at 5-month, standing at 10-month and walking at 1-year. He started speaking single words at the age of 11-month, short sentences having 2-3 words by 2 years, followed 2-step command by 4 years and was able to speak fluently. He was immunized as per schedule and was performing satisfactorily in nursery school. His social interactions were appropriate for age and he was liked by his teachers and classmates. He had an elder brother who was normal and no one in his family suffered from any neurological disease.

The patient was brought under our care after 1 month of his illness having received intravenous methyl prednisolone $(10 \mathrm{mg} / \mathrm{kg})$ for 5 days. He also received phenytoin and carbamazepine without any improvement. On examination, the patient was a right-handed child with average nutrition. His general examination was normal. Hearing was normal but comprehension to verbal commands was impaired. He understood simple gestures e.g. for eating, drinking and could name common objects. He spoke in monosyllables with a hesitant and forceful speech. His visual comprehension tested by showing him different photographs and drawings was normal. He could neatly write numbers, letters and draw simple figures with normal performance for his age. He was social and behaved appropriately. His systemic and rest of neurological examination were normal.

His blood count, hematology, urinalysis, serum

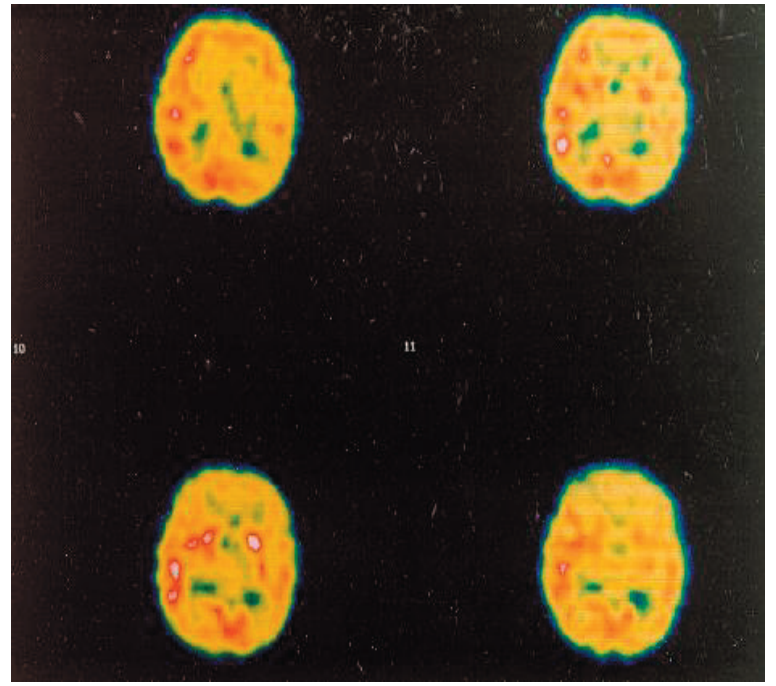

Figure 1b. ${ }^{99} \mathrm{mTc}$ ECD SPECT study of the same patient showing hypoperfusion of left fronto-temporoparietal area.

chemistry and cerebrospinal fluid (CSF) were normal. EEG in the initial stage revealed background activity of $6 \mathrm{~Hz}, 40 \mu \mathrm{V}$ without any asymmetry in voltage or frequency. There were $2-2.5 \mathrm{~Hz}$ spike and slow spike wave discharges, which were more marked during sleep. Hyperventilation and photic stimulation did not produce any change. EEG after 1 month revealed background activity of $6 \mathrm{~Hz}, 50 \mu \mathrm{V}$ with superimposed beta activity in frontocentral region with slow spike wave discharges, which are more marked on the left temporal region (Figure 1a). During sleep there was high voltage rhythmic activity. Brainstem auditory evoked potentials were normal on both sides. Cranial computerized tomography (CT) scan was normal. ${ }^{99} \mathrm{mTc}$ ECD single photon emmission tomography (SPECT) study revealed hypoperfusion in left frontotemporoparietal area (Figure 1b). On semiquantitative measurement of activities on right and left side, area of interest revealed hypoperfusion in left frontotemporoparietal areas. The right to left ratios in frontal was 3367/2966, parietal $3143 / 2770$, temporal 3119/2779 and occipital 3055/3055.

The child was prescribed phenytoin ( $50 \mathrm{mg} /$ day) and valproate (200 $\mathrm{mg}$ twice daily). At the time of discharge there was no significant change in his clinical picture. At 6 month follow up, the child became completely aphasic and started having generalised tonic clonic seizure. He was treated with intravenous immunoglobulin (IVIg) $400 \mathrm{mg}$ / $\mathrm{kg}$ for 3 days without any clinical benefit. At oneyear follow up, the child is aphasic, and was having generalised tonic clonic seizures 4-5 attack per month. The dose of sodium valproate was increased to $300 \mathrm{mg}$ twice daily and clobazam was added. At 2-year follow up although his clinical seizures were controlled but mental status remained same. 


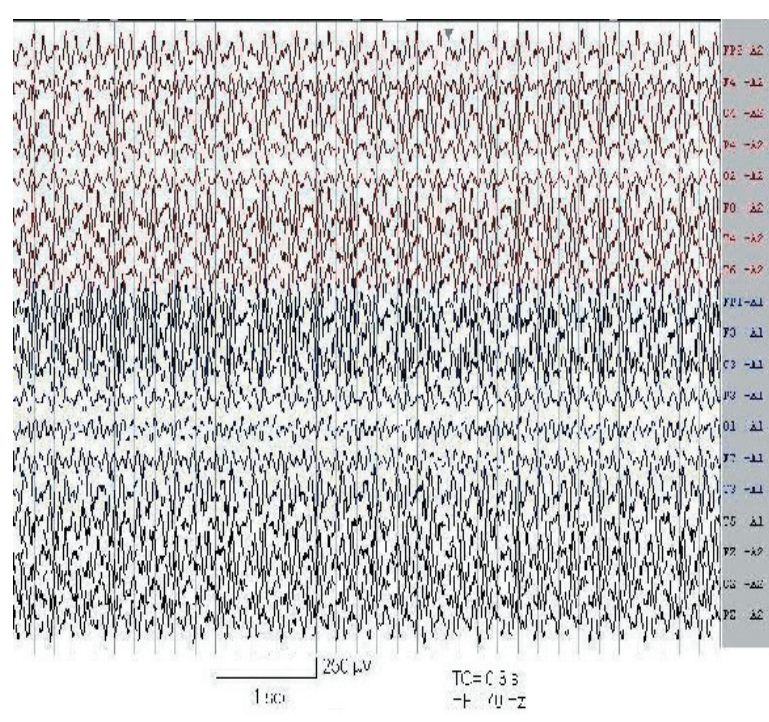

Figure 1c. EEG recording of the same patient after 2 years showed continuous spike wave discharges.

Repeated EEG both during sleep and wake showed continuous spike wave discharges (Figure 1c). Administration of intravenous diazepam did not result any change in the EEG and clinical status.

\section{Discussion}

Our patient suffered from LKS as evidenced by acquired aphasia and slow spike wave discharges, which is more marked in left temporal region. Generally LKS develops insidiously or sub acutely, however, our patient developed LKS acutely simulating a vascular accident or an encephalitic illness. However, these possibilities are unlikely because of absence of fever, other neurological signs, and normal CT scan and CSF findings.

Initially the patients with LKS develop word deafness which progresses to a total unresponsiveness to oral communication, while expressive language and vocabulary deteriorate progressively (4). In our patient, however, both auditory agnosia and expressive aphasia were noted almost simultaneously. In most patients, LKS manifests before the child has learnt reading or writing but older children who have acquired these skills may loose them (3). Our patient had very good writing and drawing skills and these were unaffected. This may be due to his excellent premorbid writing and drawing skills. The other possibility is that in a child, cerebral dominance may not develop by the age of 4.5 years; and he was only able to draw, write letters and numbers which may well be carried out by right hemisphere. On SPECT studies, his blood flow to right hemisphere was normal and EEG also showed predominant left temporal spike slow wave discharges.

SPECT and positron emission tomography (PET) studies in LKS have shown areas of decreased perfusion or metabolism in left temporal and frontoparietal area (5). Degree of regional cerebral perfusion impairment does not correlate with the clinical severity and EEG abnormality. EEG abnormalities and increased glucose metabolism in the active phase of spike wave discharges during sleep and wake stage have also been reported in LKS (6). Persistent hypometabolism or hypoperfusion may be due to enduring metabolic change (7).

Our patient did not have overt seizures in the beginning in contrast to usual clinical picture of LKS in which $80 \%$ patients have seizures which may manifest with eye blinking, brief ocular deviation, head drop, minor automatism with occasional secondary generalization. It has been suggested that LKS develops due to over abundant growth of axonal processes and synaptogenesis, which should have normally been pruned (8). Surgical specimens following subpial resection in 10 patients with LKS revealed a variety of pathologic abnormalities: subcortical astrocytosis, focal encephalitis, cryptic arteriovenous malformations, ectopic neurons and normal tissue (9). These findings are quite similar to other reports of pathological findings in the specimens examined after epilepsy surgery. LKS is a mixed bag; it is a clinical syndrome manifesting with localisation related perisylvian pathology. Landau himself has considered this syndrome as a badge of ignorance and recommended further studies on etiology, pathology and rational therapy (10). Our patient with LKS also developed protracted and severe clinical and EEG changes over 2-year follow up. In LKS, slow spike wave discharges are more prominent during sleep. In our patient also there were slow spike wave discharges during slow wave sleep; however, at 2 years follow up these discharges were present during awake period also. The syndrome of LKS and continuous slow spike wave during slow sleep (CSWS) are two polar forms of functional childhood epileptic encephalopathy (11). Symptomatology, EEG findings and course were found to overlap between Rolandic epilepsy, LKS and CSWS and these disorders may represent a continuum. Transformation of clinical semiology of LKS to CSWS has been reported in four patients and authors have suggested that duration age of onset, site of epileptiform activity and individual neurophyschological profile are useful prognostic indicators (12). In a long term follow up study of five patients with CSWS though the clinical seizures were controlled three of them remained globally and non-selectively mentally deficient. As opposed to this LKS patients had normal intellect; however life was disrupted due to severe language disturbance (13). Although the follow up EEG in our patient simulated CSWS, his intellect was preserved. Significant improvement of LKS patient following IVIg treatment has been reported (14). 
Our patient however did not improve following IVIg, which may be due to heterogeniety of the disease.

This patient with LKS reveals many uncommon features such as acute onset, preservation of drawing and writing skills, simultaneous occurrence of auditory agnosia and expressive aphasia, refractoriness to methyl prednisolone and IVIg and transition to electrical status.

\section{Acknowledgement}

We acknowledge Rakesh Kumar Nigam for technical assistance.

\section{References}

1. Morrell F, Whisler WW, Smith MC, et al. LandauKleffner syndrome. Treatment with subpial intracortical transection. Brain 1995; 118: 15291546.

2. Paquier PF, Van Dongen HR, Loonen CB. The Landau-Kleffner syndrome or, acquired aphasia with convulsive disorder. Long-term follow-up of six children and a review of the recent literature. Arch Neurol 1992; 49: 354-359.

3. Rapin I, Mattis S, Rowan AJ, Golden GG. Verbal auditory agnosia in children. Dev Med Child Neurol 1977; 19: 197-207.

4. Paquier PF, Van Dongen HR, Loonen CB. The Landau-Kleffner syndrome or, acquired aphasia with convulsive disorder. Long-term follow-up of six children and a review of the recent literature. Arch Neurol 1992; 49: 354-359.

5. Sayit E, Dirik E, Durak H, Uzuner N, Anal O, Çevik NT. Landau-Kleffner syndrome: relation of clinical, EEG and Tc-99mHMPAO brain SPECT findings and improvement in EEG after treatment. Ann Nucl Med 1999; 13: 415-418.
6. Hirsch E, Maquet P, Metz-Lutz M-N, Motte J, Finck S, Marescaux C. The eponym "Landau-Kleffner syndrome" should not be restricted to childhoodacquired aphasia with epilepsy. In: Beaumanoir A, Bureau M, Deonna T, Mira L, Tissanari CA (eds). Continuous spikes and during slow sleep electrical status epilepticus during slow sleep. London: John Libbey, 1995, pp 57-62.

7. Maquet P, Hirsch E, Metz-Lutz MN, et al. Regional cerebral glucose metabolism in children with deterioration of one or more cognitive functions and continuous spike-and-wave discharges during sleep. Brain 1995; 118: 1497-1520.

8. Smith MC. Landau-Kleffner syndrome and continuous spikes and waves during slow sleep. In: Engel J, Pedley TA (eds). Epilepsy. Philadelphia: Lippincott-Raven, 1998, pp 2367-2377.

9. Smith MC, Pierre-Louis SJC, Kanner AM, et al. Pathological spectrum of acquired epileptic aphasia of childhood. Epilepsia 1992; 33: 115 (Abstract).

10. Landau WM. Landau-Kleffner syndrome. An eponymic badge of ignorance. Arch Neurol 1992; 49: 353.

11. Smith MC, Hoeppner TJ. Epileptic encephalopathy of late childhood: Landau-Kleffner syndrome and the syndrome of continuous spikes and waves during slow-wave sleep. J Clin Neurophysiol 2003; 20: 462472.

12. Veggiotti P, Termine $\mathrm{C}$, Granocchio E, Bova S, Papalia G, Lanzi G. Long-term neuropsychological follow-up and nosological considerations in five patients with continuous spikes and waves during slow sleep. Epileptic Disord 2002; 4: 243-249.

13. Praline J, Hommet C, Barthez MA, et al. Outcome at adulthood of the continuous spike-waves during slow sleep and Landau-Kleffner syndromes. Epilepsia 2003; 44: 1434-1440.

14. Fayad MN, Choueiri R, Mikati M. LandauKleffner syndrome: consistent response to repeated intravenous gamma-globulin doses: a case report. Epilepsia 1997; 38: 489-494. 